\title{
Covid-19, diseño biomecánico del puesto de auxiliar de enfermería. Caso del hospital: Universidad Técnica particular de Loja.
}

Covid-19. Biomechanical Design of the Nursing Assistant post. Hospital case: Private Technical University of Loja

Rita Elizabeth Velasteguí Hernandez. ${ }^{1}$, Manolo Alexander Córdova Suárez. ${ }^{2}$ \& Edison Patricio Villacres Cevallos. ${ }^{3}$

\begin{abstract}
.
Resumen.

Introduction. The coronavirus pandemic has caused a lot of damage not only to the people it affects but also to the health professionals who care for them. The activities carried out by the Nursing Assistants (ADE) have exposure to static charges that can cause affections to the osteo myoarticular system. Objective. The manipulation activity of patients in established positions complying with Covi-19 medical care procedures in the

Introducción. La pandemia por coronavirus ha causado mucho daño no solo a las personas que afecta sino también a los profesionales de salud que los atienden. Las actividades que realizan los Auxiliares de Enfermería (ADE) tienen exposición a cargas estáticas que pueden ocasionar afecciones al sistema osteo mioarticular. Objetivo. Se estudió ergonómicamente la actividad de manipulación de pacientes en posiciones

1 Regional Autonomous University of Los Andes, Postgraduate, Ambato, Ecuador, pg.ritaevh88@uniandes.edu.ec, (D) https://orcid.org/0000-0001-9360-4265

2 Universidad Nacional de Chimborazo, Facultad de Ingeniería, Riobamba-Ecuador, manolo.cordova@unach.edu.ec (D) https://orcid.org/0000-0001-6786-7926

3 Universidad Nacional de Chimborazo, Facultad de Ingeniería, Riobamba-Ecuador, pvillacres@ unach.edu.ec io https://orcid.org/0000-0001-9518-1278
\end{abstract}


Intensive Care Units (ICU) of the Hospital Universidad Técnica Particular de Loja (UTPL) was ergonomically studied, using the BIO-mec computer program. Methodology. It began with the observational identification of: a) activities, b) tasks, c) times and movements that the ADE executes when handling the patient in the supine and prone position due to improved ventilation and evacuation of pulmonary fluids, in ICUs Hospital UTPL that can cause excess of the worker's body limits in the osteo myoarticular system. Then, a calculation was made of the percentage of the maximum load, overload, bearable risk achieved in each joint established by the BIO-mec method before and after the implementation of a hospital bed adjustable to the vertical height of the nursing assistant. Results. The improvements of the position show a negative effect on the maximum admissible loads of the elbow, shoulder, torso, hip, knee and ankle, improving the posture angles of: $130^{\circ}$ for knee, $94^{\circ}$ hip, $50^{\circ}$ for L5 / S1, $40^{\circ}$ for shoulder, $130^{\circ}$ for elbow and $90^{\circ}$ wrist for men, improving the 6 postural load elements. For women, the implementation improved the posture angles of: $130^{\circ}$ for knee, $94^{\circ}$ hip, $50^{\circ}$ for L5 / S1, $40^{\circ}$ for shoulder, $130^{\circ}$ for elbow and $90^{\circ}$ wrist, improving all 4 of the 6 load elements. postural. Conclusion. The use of a vertical height adjustable patient bed improves the posture of the nursing assistant, reducing $66.66 \%$ of the ergonomic affectation for women and $100 \%$ for men.

Keywords: Nursing assistant, biomechanics, hospital bed, BIO-mec. establecidas que cumplen procedimientos de atención médica por Covi-19 en las Unidades de Cuidado Intensivo (UCI) del Hospital Universidad Técnica Particular de Loja (UTPL), utilizando el programa informático BIO-mec. Metodología. Se empezó con la identificación observacional de: a) las actividades, b) tareas, c) tiempos y movimientos que ejecuta el ADE al manejar el paciente a posición decúbito supino y decúbito prono por mejora de ventilación $\mathrm{y}$ desalojo de fluidos pulmonares, en las UCI del Hospital UTPL que pueden causar exceso de los límites corporales del trabajador en el sistema osteo mioarticular. Luego se efectuó un cálculo del porcentaje de la carga máxima, sobrecarga, riesgo soportable alcanzado en cada articulación que establece el método BIO-mec antes y después de la implementación de una cama hospitalaria ajustable a la altura vertical del auxiliar de enfermería. Resultados. Las mejoras del puesto muestran afectación negativa en las cargas máximas admisibles de codo, hombro, torso, cadera, rodilla y tobillo, mejorando los ángulos de postura de: $130^{\circ}$ para rodilla, $94^{\circ}$ cadera, $50^{\circ}$ para $\mathrm{L} 5 / \mathrm{S} 1,40^{\circ}$ para hombro, $130^{\circ}$ para codo y $90^{\circ}$ muñeca para los hombres, mejorando los 6 elementos de carga postural. Para las mujeres la implementación mejoró los ángulos de postura de: $130^{\circ}$ para rodilla, $94^{\circ}$ cadera, $50^{\circ}$ para $\mathrm{L} 5 / \mathrm{S} 1,40^{\circ}$ para hombro, $130^{\circ}$ para codo y $90^{\circ}$ muñeca mejorando los 4 de los 6 elementos de carga postural. Conclusión. El uso de una cama de paciente ajustable a la altura vertical mejora la postura del auxiliar de enfermería disminuyendo el 66,66\% de la 
afectación ergonómica para las mujeres y el $100 \%$ para los hombres.

Palabras claves: Auxiliar de enfermería, biomecánica, cama hospitalaria, BIOmec.

\section{Introducción.}

La pandemia por coronavirus es una enfermedad infecciosa causada por el COVID-19 que ha generado demasiados problemas no solo a las personas contagiadas sino también al personal de salud que se involucra en su tratamiento(Ahmed, Ramadan, Refay, Khashbah, \& neurosurgery, 2021). Muchas de las personas que sufren esta enfermedad determinan el uso de instrumentos para soporte vital en lugares especiales denominados Unidades de Cuidados Intensivos, con la asistencia de personal especializado que ejecuta actividades de levantamiento, traslado y manipulación de pacientes que exigen los niveles normales de aplicación, asimiento de fuerzas generadas por la repetitividad, duración de las posturas y pesos que sujeta el personal de salud (Hoogendoorn et al., 2021) y causan afectación en su bienestar físico.

El personal de salud que da soporte en estas actividades, maneja de forma manual el peso de los pacientes, pudiendo producirse lesiones en muchas zona del cuerpo, siendo más recursivas de afectación los miembros superiores, espalda y principalmente la zona lumbar (Sanz et al., 2020).

Existe un elevado número de enfermeras y auxiliares de enfermería que refieren molestias a nivel lumbar debido al manejo de pacientes en UCI que requieren cambio de posturas para lograr una mejora de la ventilación y desalojo de fluidos pulmonares en los pacientes (Aguilar \& Domínguez, 2021).

El diseño de puestos de trabajo introduce el concepto de ergonomía para atenuar la exposición a factores de riesgo relacionados con el manejo de carga estáticas y posturas de trabajo continuas. Esta manipulación que se realiza de una manera rápida y sin entrenamiento agrava la situación del auxiliar de enfermería que se ve forzado a ejecutar actividades de manera inmediata para evitar el contagio, forzando ciertas partes del cuerpo por la ejecución de estas tareas (Suárez, Vasco, Ochoa, \& Puca, 2020). Para mitigar los efectos disergonómicos causados por estas sobrecargas y que afectan al sistema osteo mioarticular se debe rediseñar los puestos de trabajo con fundamentos de Biomecánica o simplemente incluir nuevas ayudas mecánicas que logren la adaptación del auxiliar de enfermería en el manejo y postura de los pacientes que permanecen inmóviles (Ávila Torres, 2013).

Estas medidas preventivas ayudan en la disminución de los factores de riesgo ergonómicos pero la mayoría de ocasiones no se pueden efectuar ya que si el rediseño involucra cambios en la organización el trabajo o modificaciones costosas los directivos ven estas modificaciones como incrementos en sus egresos y complicaciones en su 
logística de trabajo por lo que se necesita simular los entornos hasta conseguir una mejora significativa probando las mejores alternativas de cambio en el puesto (Broseta, de Moya, Corresa, Jaén, \& i Pérez, 2010).

Los auxiliares de enfermería que manejan pacientes con Covid-19 en las Unidades de Cuidados Intensivos llegan a soportar cargas compartidas entre mínimo dos personas de: $40 \mathrm{~kg}$ hasta $70 \mathrm{~kg}$ desde camillas, sillas de ruedas o en el mismo sitio a las posiciones decúbito supino y decúbito prono (Montaguano \& Jiménez, 2021) causando desordenes osteo mioarticulares y hasta accidentes con caída de pacientes o lesiones en el personal de salud que interviene en estas actividades (Jurado Colmena, 2021).

En la actualidad, el uso de simulación ha aumentado de una manera importante su participación ya que garantiza resultados antes de la ejecución real, evitando gastos por malos diseños y pérdidas económicas trascendentales (Franco Ulloa, 2018). El método BIO-mec calcula el porcentaje de la carga máxima, sobrecarga, riesgo soportable alcanzado en cada articulación lo que ayuda al diseño ergonómico hasta lograr algo óptimo (Urquizo Manrique \& Bedoya García, 2019).

Esta investigación utiliza la ergonomía de diseño de los puestos de trabajo con el uso de la biomecánica para la mejora de puestos muy recurrentes y tan importantes en estas condiciones de pandemia.

\section{Metodología}

\section{Diseño Biomecánico de puestos de trabajo.}

El diseño ergonómico garantiza una distribución correcta de los espacios y brinda una disposición armónica de los medios (Mondelo, Bombardo, Busquets, \& Torada, 2004). Además, proporciona una mejor dinámica en la ejecución de esfuerzos y movimientos para un mejor desempeño del trabajo diario (Delgado Vilca \& Puma Flores, 2020). La biomecánica es una especialidad de la ergonomía que ayuda a una mejor ubicación de las posiciones resultantes al ejecutar movimientos complejos, mejora la comodidad y atenúa esfuerzos (Lucero Guerrero, 2014). Por otro lado, estos métodos biomecánicos se basan en datos relativos a características funcionales de las personas y su aplicación y estudios implican gastos elevados para los empleadores; dejando como alternativa el uso de soluciones virtuales con software que utiliza modelos biomecánicos e inteligencia artificial (Jorge, 1999).

\section{Método BIO-mec.}

Uno de los métodos de análisis biomecánico más utilizados es la herramienta BIO-mec (Mira, Cardona, Vargas, \& Buitrago, 2020) que fue desarrollado por la Universidad Politécnica de Valencia (Pacheco Cruz \& Suárez Rativa) estudia el comportamiento de manera física calculando la carga estática postural recomendada y crítica para cada parte del cuerpo sujeta a tensión y torsión por la ejecución a las tareas de manera estática (Remesal, 2004). 
El método BIO-mec establece una analogía entre el sistema hombre-máquina, utiliza un bípedo (Ver figura1.) compuesto por palancas y poleas para evaluar el esfuerzo de una posición dada en causar sobrecarga en alguna estructura del aparato locomotor (Jaramillo Muñoz \& Londoño Pérez, 2020).

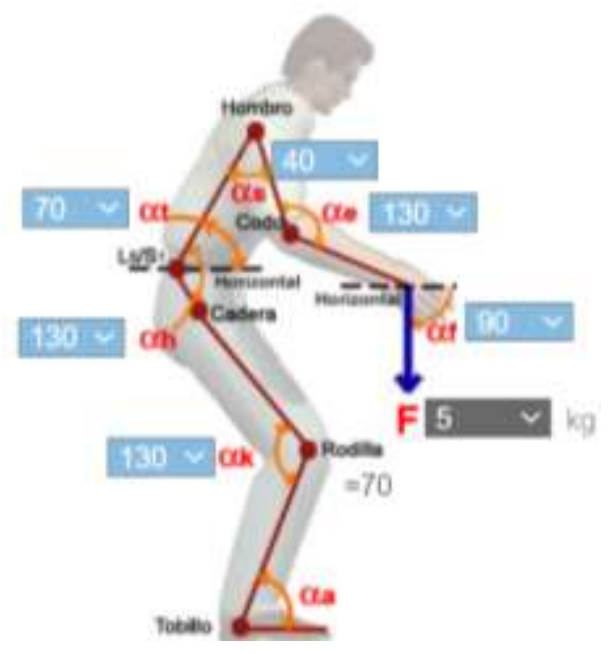

Figura 1. Bípedo de ingreso para ángulos y posturas con BIO-mec. Nota: Tomado de software en línea www.ergonaurtas.upv.es

El BIO-mec realiza un estudio de dinámica y estática de fuerzas con el uso de ecuaciones de equilibrio para conocer: a) los momentos y b) las fuerzas de contrarrestre de las cargas que sujetan los miembros del cuerpo divididos en secciones (Ridaura, de Rosario Martínez, Gascón-Pelegrí, \& Barrachina, 2007). Cada articulación se considera punto de apoyo. Para su aplicación requiere de datos propios del trabajador que ocupa el puesto de trabajo como: a) género, b) tipo de soporte de la carga, c) Peso del trabajador, d) estatura del trabajador, e) Tiempo de duración de la postura, f) repetitividad de la postura, g) Ángulos entre segmentos, h) coeficiente de rozamiento calzado/suelo, h) percentil de protección.

Como resultado el BIO-mec arroja: el porcentaje de la carga máxima soportable alcanzado en cada articulación (Codo, hombro, torso, cadera, rodilla, tobillo) con una indicación de alarma al sobrepasar el límite recomendable. También calcula las cargas máximas, sobrecarga, riesgo para: a) codo, b) hombro, c) lumbar (L\%/S1), d) cadera, e) rodilla, f) tobillo. Como último resultado arroja los pesos y longitud de los siguientes miembros: a) cabeza, b) cuello, c) tórax, d) abdomen, e) pelvis, f) brazo, g) antebrazo, h) mano, i) muslo, j) pierna, y k) pierna (Saavedra-Robinson, Marín-Londoño, \& PalaciosGonzález, 2018).

\section{Rediseño biomecánico}

Se estudió las dos posiciones referidas como críticas en el puesto de Auxiliar de Enfermería cambiando las variables más importantes: peso del trabajador, estatura del trabajador, ángulos de postura y peso del paciente (Alvis, Rodríguez, ORTIZ, \& Hernández, 2019). 


\section{Resultados y discusión}

\section{Resultados de la observación de campo}

Al analizar con el método observacional el puesto de trabajo de Auxiliar de Enfermería se encontró los siguientes resultados (Ver Tabla 1)

Tabla 1. Datos del puesto de trabajo

\begin{tabular}{|c|c|c|}
\hline ITEM & Elemento & Observación \\
\hline \multirow{4}{*}{ Datos del puesto } & Nombre del puesto & $\begin{array}{l}\text { Auxiliar de } \\
\text { enfermería }\end{array}$ \\
\hline & $\begin{array}{l}\text { Repetitividad y duración de la } \\
\text { postura }\end{array}$ & Menos de una hora \\
\hline & Postura analizada & Se lleva a cabo rara \\
\hline & & vez \\
\hline \multirow[b]{2}{*}{ Condiciones adicionales } & Coeficiente de rozamiento & $0,8^{\mathrm{a}}$ \\
\hline & $\begin{array}{c}\text { Porcentaje de la población a } \\
\text { proteger }\end{array}$ & $90 \% \mathrm{~b}$ \\
\hline
\end{tabular}

Luego se introduce los datos de la postura crítica (Izurieta Burgos, 2016) ver la Fig. 2.

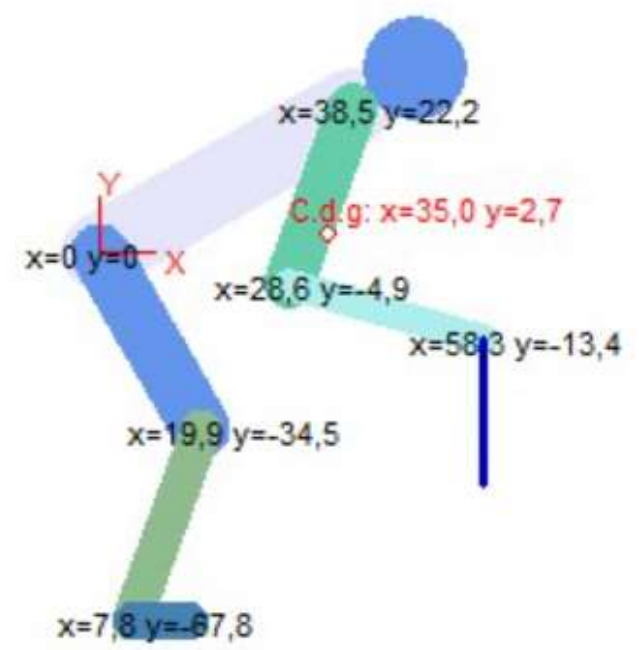

Figura 2. Postura crítica Auxiliar de enfermería en UCI Nota: El peso de la carga se repartió en dos personas simétricamente.

\section{Resultados del método BIO-mec en condiciones iniciales}

Se observa los resultados del porcentaje de cargas máximas en condiciones iniciales (Ver la Fig. 3.) 


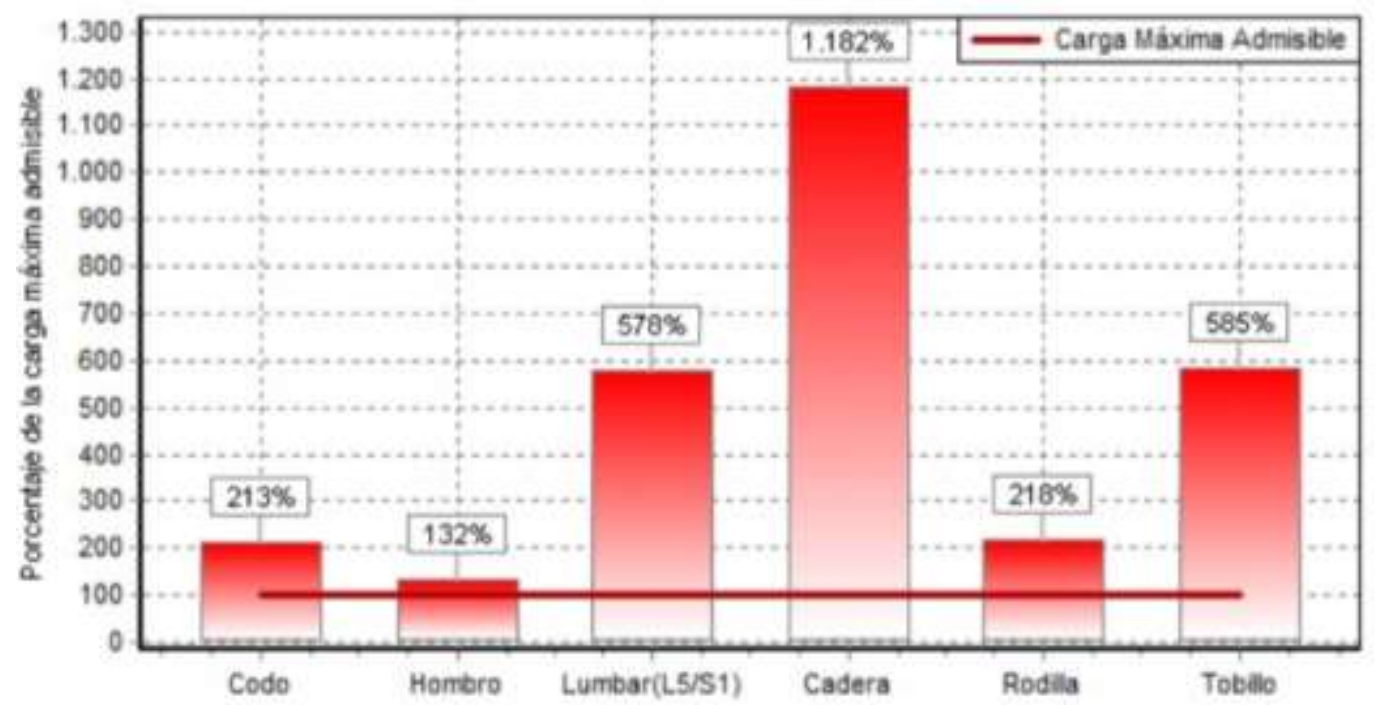

Figura 3. Porcentaje de la carga máxima. Auxiliar de enfermería Nota: Adaptado BIO-mec. Se consideró género femenino.

Para género masculino se observa los resultados del porcentaje de cargas máximas en condiciones iniciales en la Fig. 4.

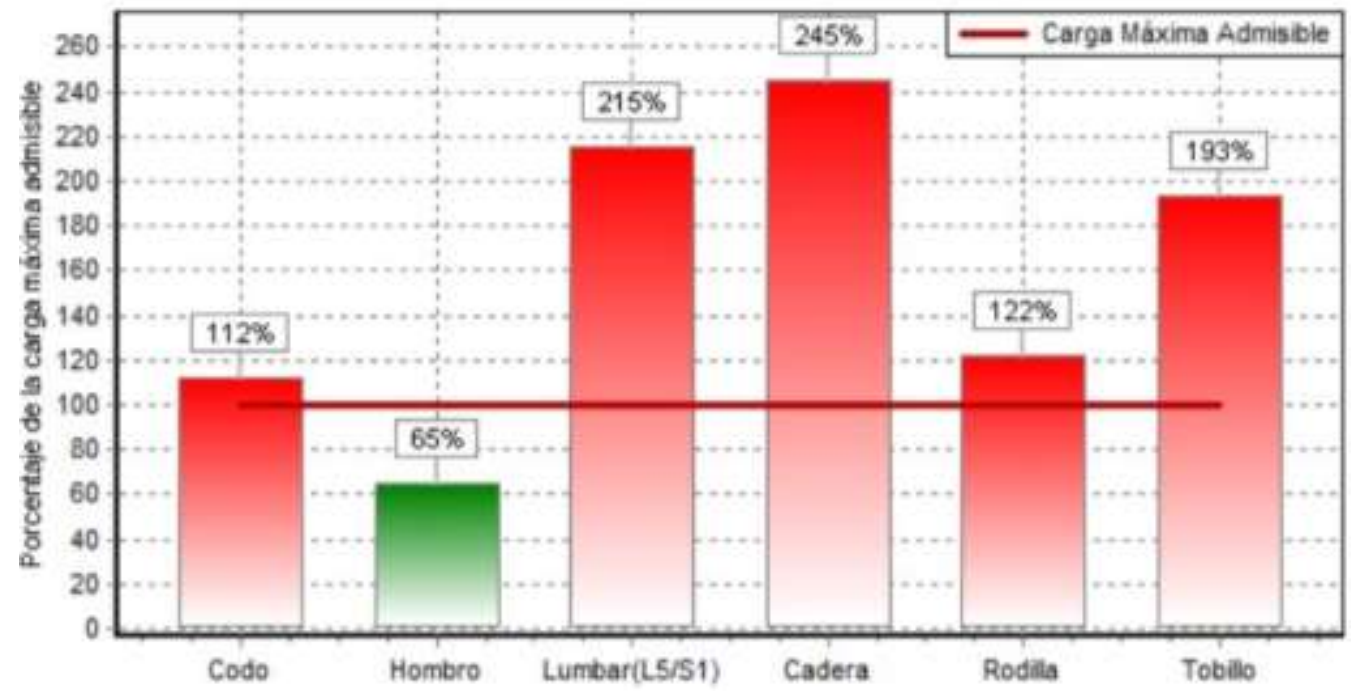

Figura 4. Porcentaje de la carga máxima. Auxiliar de enfermería Nota: Adaptado BIO-mec. Se consideró género masculino.

\section{Resultados del método BIO-mec en condiciones de mejora.}

Se consideró el uso de una cama del paciente ajustable a la altura antropométrica del Auxiliar de Enfermería determinando la postura de la figura 5. 

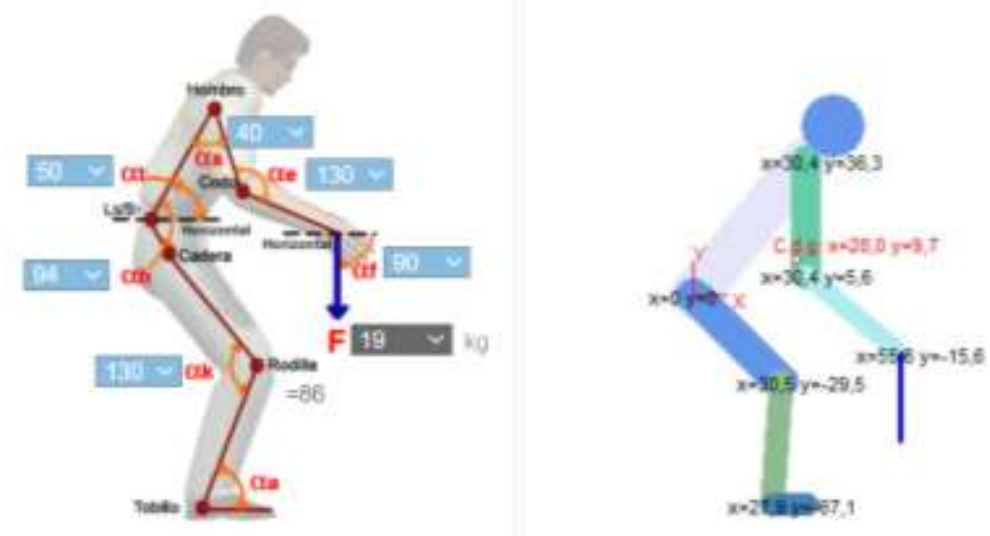

Figura 2. Postura crítica Auxiliar de enfermería en UCI (mujer) Nota: El peso de la carga se repartió en tres personas simétricamente.

Se consideró el uso de una cama del paciente ajustable a la altura antropométrica del Auxiliar de Enfermería determinando la postura de la figura 5.
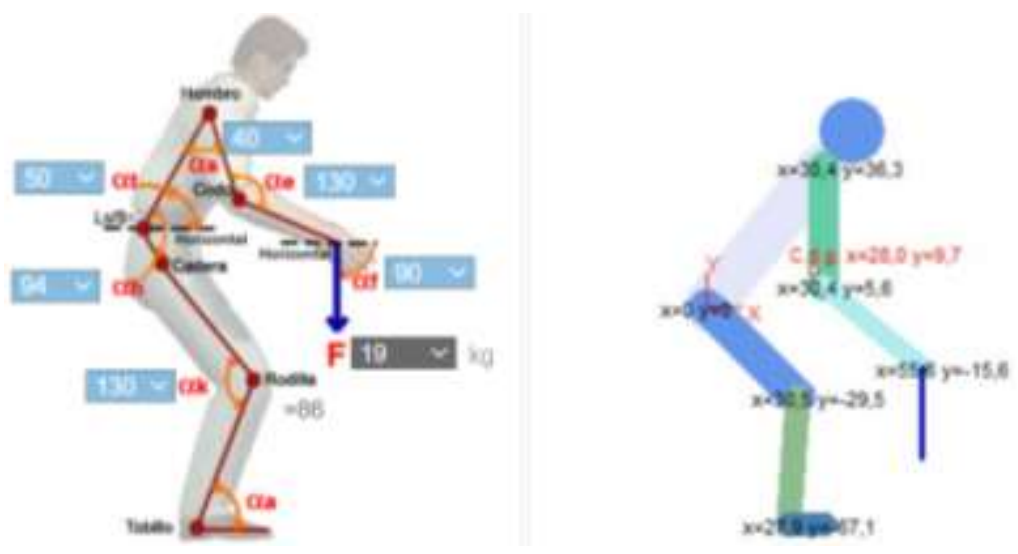

Figura 5. Postura recomendada Auxiliar de enfermería en UCI (hombre)

Nota: El peso de la carga se repartió en tres personas simétricamente. Adaptado de www.ergonautas.upv.es

Luego de establecer la carga máxima $(57 \mathrm{~kg}$ ) del paciente y considerar los ángulos de la posición que determina el rediseño se observa los siguientes resultados para trabajador mujer. Ver figura 6.
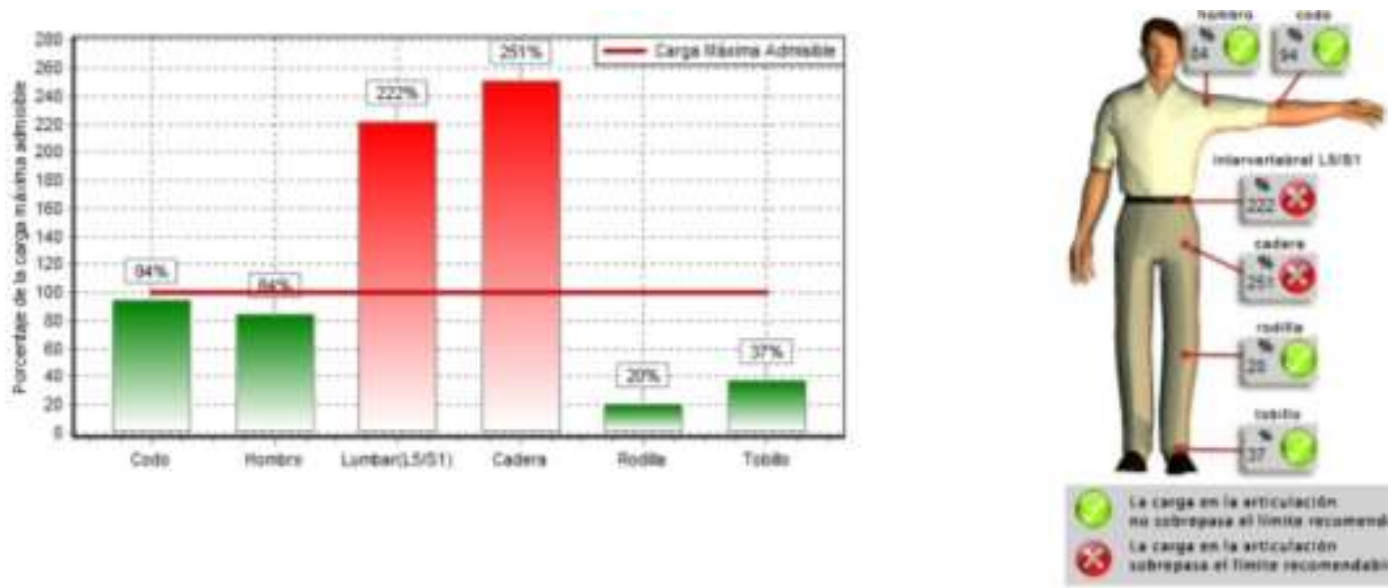

Figura 6. Porcentaje de carga máxima soportad. Auxiliar de enfermería en UCI (mujer) Nota: El peso de la carga se repartió en tres personas simétricamente. Adaptado de www.ergonautas.upv.es 
Luego de establecer la carga máxima $(57 \mathrm{~kg}$ ) del paciente y considerar los ángulos de la posición que determina el rediseño se observa los siguientes resultados para trabajador hombre. Ver figura 7.
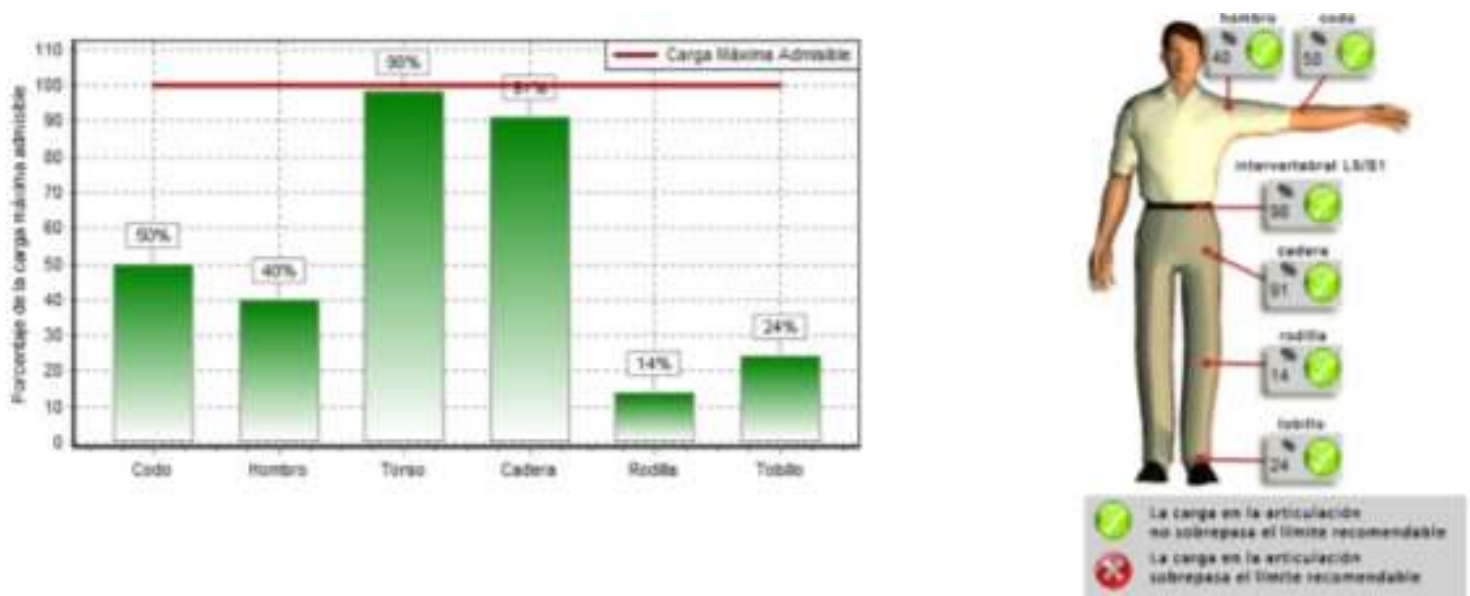

Figura 7. Porcentaje de carga máxima soportad. Auxiliar de enfermería en UCI (hombre) Nota: El peso de la carga se repartió en tres personas simétricamente. Adaptado de www.ergonautas.upv.es

\section{Conclusiones}

- Con el uso de una cama de paciente ajustable y al reducir un 18,57 \% el peso de la carga máxima para mujer en el puesto de trabajo de Auxiliar de enfermería y con ángulos de: $130^{\circ}$ para rodilla, $94^{\circ}$ cadera, $50^{\circ}$ para $\mathrm{L} 5 / \mathrm{S} 1,40^{\circ}$ para hombro, $130^{\circ}$ para codo, $90^{\circ}$ muñeca se redujo las cargas máximas de: codo, hombro, rodilla y tobillo a los límites recomendados quedando: lumbar $122 \%$ y cadera con un $151 \%$ sobre el límite recomendado. Por otro lado, con el uso de una cama de paciente ajustable y al reducir un $18,57 \%$ el peso de la carga máxima para hombre en el puesto de trabajo de Auxiliar de enfermería y con ángulos de: $130^{\circ}$ para rodilla, $94^{\circ}$ cadera, $50^{\circ}$ para $\mathrm{L} 5 / \mathrm{S} 1,40^{\circ}$ para hombro, $130^{\circ}$ para codo, $90^{\circ}$ muñeca se redujo las cargas máximas de: codo, hombro, rodilla, tobillo lumbar y cadera a los límites recomendados.

\section{Referencias bibliográficas.}

Aguilar, Á. R., \& Domínguez, P. L. J. E. D. (2021). Cuidados al paciente COVID en una unidad de cuidados intensivos. 56-61.

Ahmed, G. K., Ramadan, H. K.-A., Refay, S. M., Khashbah, M. A. J. T. E. j. o. n., psychiatry, \& neurosurgery. (2021). Comparison of knowledge, attitude, socioeconomic burden, and mental health disorders of COVID-19 pandemic between general population and health care workers in Egypt. 57(1), 1-11.

Alonso Montero, C. (2009). Calzado de seguridad y el papel del podólogo en la salud laboral. Universidad Miguel Hernández, 
Alvis, N. C. R., Rodríguez, J. M. T., ORTIZ, S. P. Q. P., \& Hernández, P. N. O. J. R. I. (2019). Riesgos biomecánicos en los funcionarios del Instituto de Educación a Distancia de la Universidad del Tolima, periodo 2016-2019/Fuero de Salud. 9(1).

Ávila Torres, D. E. (2013). Estudio ergonómico y rediseño en puesto de trabajo para el sector del calzado.

Broseta, M. J. V., de Moya, M. F. P., Corresa, S. P., Jaén, J. D. G., \& i Pérez, E. S. J. R. d. b. (2010). Nuevo servicio de valoración biomecánica de los accidentados de tráfico. Caso clínico: diagnóstico de la simulación. (53), 11-14.

Delgado Vilca, G. J., \& Puma Flores, K. R. (2020). Ergonomía y desempeño laboral en la gerencia de administración de la EPS SEDACUSCO SA 2020.

Franco Ulloa, J. H. (2018). Plan de prevención de riesgos físicos, mecánicos y ergonómicos por puesto de trabajo en la Empresa Optec. Universidad de Guayaquil. Facultad de Ingeniería Industrial. Carrera de ...,

Hoogendoorn, M. E., Brinkman, S., Bosman, R. J., Haringman, J., de Keizer, N. F., \& Spijkstra, J. J. (2021). The impact of COVID-19 on nursing workload and planning of nursing staff on the Intensive Care: A prospective descriptive multicenter study. International Journal of Nursing Studies, 121. doi:10.1016/j.ijnurstu.2021.104005

Izurieta Burgos, L. K. (2016). Diseño de controles operacionales y evaluaciones ergonómicos de las actividades de recolección de basura del GAD Municipal de Pedro Carbo 2014" Guayaquil". Universidad de Guayaquil. Facultad de Ingeniería Industrial. Maestría en ...,

Jaramillo Muñoz, S., \& Londoño Pérez, E. (2020). Propuesta de un método de apoyo a la ergonomía para el sector floricultor, mediante el uso de dispositivos y tecnologías.

Jorge, R. L. (1999). Diagnóstico funcional de registros biomecánico s de marcha humana mediante técnicas de ingeniería de sistemas y de inteligencia artificial. Contribución a la valoración objetiva de la discapacidad. Universitat Politècnica de València,

Jurado Colmena, B. (2021). Manejo de paciente intubado con covid 19, por profesional quirúrgico de enfermería en la Unidad de Terapia Intensiva del Hospital Obrero\# 30, tercer trimestre, 2020.

Lucero Guerrero, P. F. (2014). Diseño, experimentación y evaluación de prácticas en el área de ergonomía, modelado biomecánico y análisis de movimiento para un Laboratorio de Ingeniería Biomédica en la Universidad Politécnica Salesiana sede Cuenca. 
Mira, N. O., Cardona, I. C. S., Vargas, K. C. C., \& Buitrago, P. A. R. (2020). Biomechanics of the dorsolumbar region during manual patient handling. Ciencia e Innovación en Salud.

Mondelo, P. R., Bombardo, P. B., Busquets, J. B., \& Torada, E. G. (2004). Ergonomía 3: Diseño de puestos de trabajo (Vol. 3): Universitat Politecnica de Catalunya. Iniciativa Digital Politecnica.

Montaguano, J. A. V., \& Jiménez, E. I. A. J. E. I. (2021). PERCEPCIÓN DEL PROFESIONAL DE ENFERMERÍA SOBRE LOS CUIDADOS APLICADOS AL PACIENTE EN POSICIÓN DE DECUBITO PRONO ASOCIADO AL COVID-19. 6(2), 36-42.

Pacheco Cruz, K. J., \& Suárez Rativa, K. L. Identificación y evaluación de factores de riesgo biomecánico en operarios de inhumación, exhumación y horno crematorio de inversiones el Paraíso parque cementerio.

Remesal, A. F. J. R. d. b. (2004). Análisis ergonómico de puestos de trabajo en el sector de calzado. (42), 27-30.

Ridaura, R. P., de Rosario Martínez, H., Gascón-Pelegrí, J. Z., \& Barrachina, N. S. J. R. d. b. (2007). Nuevas metodologías para el análisis de la usabilidad en productos de la vida diaria para personas mayores. (48), 21-23.

Saavedra-Robinson, L. A., Marín-Londoño, V., \& Palacios-González, C. J. R. U. I. (2018). Diseño de un plan de acción para reducir la carga física biomecánica en empresas del sector del calzado del Valle del Cauca. 17(2), 241-252.

Sanz, M. B., Hernández-Tejedor, A., Estella, Á., Rivera, J. J., de Molina Ortiz, F. G., Camps, A. S., . . Laguna, L. B. J. M. i. (2020). Recomendaciones de «hacer» y «no hacer» en el tratamiento de los pacientes críticos ante la pandemia por coronavirus causante de COVID-19 de los Grupos de Trabajo de la Sociedad Española de Medicina Intensiva, Crítica y Unidades Coronarias (SEMICYUC). 44(6), 371-388.

Suárez, M. A. C., Vasco, L. J. T., Ochoa, Z. E. Z., \& Puca, J. P. M. J. C. (2020). Diseño biomecánico del puesto de trabajo de noqueo en el faenamiento de ganado. 3(3.2), 6-17.

Urquizo Manrique, M. C., \& Bedoya García, S. R. (2019). Biomecánica de riesgo disergonómico y su aporte en la aparición de trastornos músculo-esqueléticos en los estibadores de la empresa Ejecutores y Consultores Bencaingenieros EIR L, Arequipa 2018.

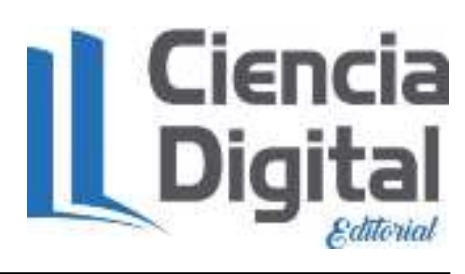




\section{PARA CITAR EL ARTÍCULO INDEXADO.}

Velasteguí Hernandez, R. E., Córdova Suárez, M. A., \& Villacres Cevallos, E. P. (2021). Covid-19, diseño biomecánico del puesto de auxiliar de enfermería. Caso del hospital: Universidad Técnica particular de Loja . Anatomía Digital, 4(3.1), 6-17. https://doi.org/10.33262/anatomiadigital.v4i3.1.1850

\section{Ciencia \\ Ligital}

El artículo que se publica es de exclusiva responsabilidad de los autores y no necesariamente reflejan el pensamiento de la Revista Anatomía Digital.

El artículo queda en propiedad de la revista y, por tanto, su publicación parcial y/o total en otro medio tiene que ser autorizado por el director de la Revista Anatomía Digital.
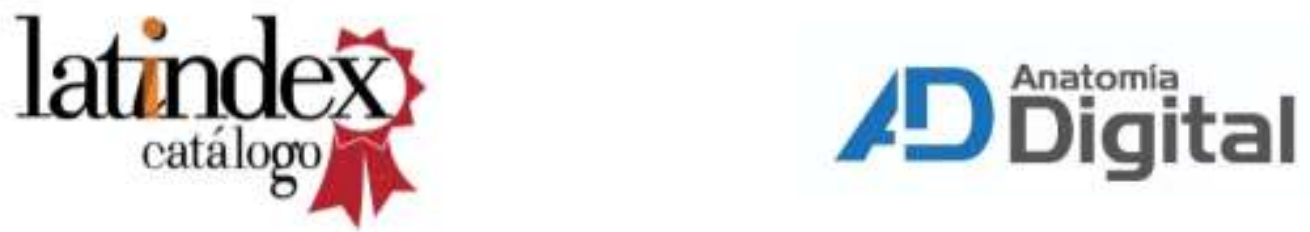